\title{
Rapid identification of Candida albicans, $C$. glabrata, C. parapsilosis and $C$. krusei by species- specific PCR of large subunit ribosomal DNA
}

\author{
${ }^{*} K$. A. HAYNES and THEA J. WESTERNENG \\ Department of Medical Microbiology, Charing Cross and Westminster Medical School, St Dunstan's Road, \\ London W6 8RF
}

\begin{abstract}
A rapid PCR-based method for the identification of four Candida species is described. Primers to conserved sequences in the V3 region of large subunit rDNA were used to amplify DNA from $C$. albicans, $C$. glabrata, $C$. parapsilosis and $C$. krusei. The sequences were aligned and areas of non-concordance were used to design four species-specific forward primers. In a blind study of 82 yeast strains, four PCRs based on these primers correctly identified nine $C$. albicans, $18 C$. glabrata, $13 C$. parapsilosis and $18 C$. krusei strains after gel electrophoresis of amplified DNA. Furthermore, of 17 other Candida strains (10 species) and seven strains from other yeast genera (Saccharomyces, Cryptococcus and Trichosporon) only one false positive amplification product (with $C$. dubliniensis) was seen. These PCRs offer a rapid alternative to conventional techniques for the identification of $C$. albicans, $C$. glabrata, $C$. parapsilosis and $C$. krusei.
\end{abstract}

\section{Introduction}

Members of the fungal genus Candida are amongst the most common hospital pathogens and are a particular problem in the management of immunocompromised patients [1]. Individuals who are infected with the human immunodeficiency virus, or undergoing bone marrow or solid organ transplantation, receiving corticosteroids or with indwelling catheters are especially at risk [2]. While $C$. albicans remains the most commonly encountered pathogen, a large number of other species, especially $C$. glabrata, C. parapsilosis and $C$. krusei, are emerging as important opportunist pathogens [3].

The antifungal susceptibility patterns exhibited by these different species are diverse [4]. Therefore, to initiate prompt antifungal therapy, a quick identification system is required. Currently, the identification of Candida spp. in the routine diagnostic laboratory relies primarily on culture of the organism and biochemical assimilation tests [5,6]. The API 20C (bioMérieux, Basingstoke, Hampshire) is a reliable

Received 6 July 1995; revised version accepted 4 Oct. 1995.

* Present address and address for correspondence: Infectious Diseases and Bacteriology, RPMS, Hammersmith Hospital, Du Cane Road, London W12 ONN. and proven system in this context. Unfortunately, only $73.4 \%$ of yeasts are identifiable after incubation for $24 \mathrm{~h}$. This rises to $99.3 \%$ after $48 \mathrm{~h} \mathrm{[5]}$.

An alternative approach to the use of biochemical assimilation tests has been the development of differential culture media that facilitate the isolation and identification of clinically important Candida spp. Several of these, e.g., Nickerson's media, rely on the reduction of bismuth salts, but they often lack discrimination, especially in the presence of bacteria [7]. However, the recent evaluation of a commercially available chromogenic medium, CHROMagar Candida (CHROMagar Co., Paris, France), demonstrated its ability to identify presumptively members of three species: C. albicans, C. krusei and C. tropicalis. The sensitivities and specificities of $>99 \%$ obtained were made on the basis of colony morphology and colour development [8]. Furthermore, this medium was able to differentiate these species directly from clinical specimens without the need for prior isolation. Unfortunately, the reported accuracy was achieved only after $48 \mathrm{~h}$ as 'colony colour development was inconsistent after $24 \mathrm{~h}$ of incubation' [8].

This delay in the identification of Candida spp. by biochemical and cultural procedures has led to a search for more rapid techniques and, recently, 
molecular methods have been applied to the epidemiology and identification of this genus $[9,10]$. Southern analysis has been employed in the identification of many fungi and commercial kits for a number of species are now available [11].

The speed and sensitivity of PCR make it an ideal choice for the basis of a rapid identification system. Although several Candida genes have been developed as targets for PCR they have generally been used to detect only C. albicans [12-14]. More recently, reports have appeared which indicate that PCRs, based on variable sequences within the small subunit rRNA genes, can be used to discriminate between $C$. albicans and other pathogenic Candida spp. $[15,16]$. Unfortunately, additional Southern hybridisation or restriction enzyme digestion, or both, are often required to identify yeasts to the species level $[9,17]$.

An alternative target that has been exploited for the development of Candida-specific PCRs is the cytochrome P-450-dependent lanosterol- $\alpha$-demethylase gene [18]; portions of this gene from $C$. glabrata and $C$. krusei were cloned and sequenced. These sequences were compared with published data for $C$. albicans and C. tropicalis, resulting in the design of four apparently species-specific primers. Experimental testing of these four primers confirmed their specificity. However, these primers had a major drawback with regard to rapid identification; they all required different cycling conditions and, therefore, could not be performed simultaneously unless four PCR machines were available.

This report describes a series of PCRs, based on sequences in the large subunit rRNA gene, that can all be performed under the same cycling conditions. These PCRs allow the rapid $(<3 \mathrm{~h})$ identification of C. albicans, C. glabrata, C. parapsilosis and $C$. krusei. This represents a saving of up to $72 \mathrm{~h}$ on currently employed identification procedures.

\section{Materials and methods}

\section{Organisms and growth conditions}

The 82 strains used in this study were clinical isolates and strains from culture collections representing 14 Candida spp. and three other genera (Table 1). Fungi were obtained from: the National Collection of Pathogenic Fungi, PHLS Regional Mycology Laboratory, Bristol; the Department of Medical Microbiology, University of Wales College of Medicine, Cardiff; the Department of Infectious Diseases and Bacteriology, Royal Postgraduate Medical School, London; the Department of Infectious Diseases and Tropical Medicine, Monsall Hospital, Manchester and the Department of Microbiology, Trinity College, University of Dublin, Ireland.
The yeasts were grown routinely on glucose $1 \% \mathrm{w} / \mathrm{v}$, yeast extract $1 \% \mathrm{w} / \mathrm{v}$ (YE) plates at $37^{\circ} \mathrm{C}$ or $30^{\circ} \mathrm{C}$ for $24-48 \mathrm{~h}$. Long-term storage was in water cultures at room temperature.

\section{Fungal DNA extraction}

For DNA extraction, yeasts stored in water cultures were subcultured on to YE plates and incubated as above. A single colony was then subcultured and DNA was extracted from this culture.

A rapid DNA extraction procedure $(<1 \mathrm{~h})$ was developed to minimise the time required for species identification. A single yeast colony was inoculated into a $1.5-\mathrm{ml}$ screw cap Eppendorf tube containing $500 \mu \mathrm{l}$ of lysis buffer $(10 \mathrm{mM}$ Tris- $\mathrm{HCl}, \mathrm{pH} 7.5,50 \mathrm{mM}$ EDTA, pH 7.5, SDS $0.2 \%$ w/v), $250 \mu \mathrm{l}$ of lysis buffer equilibrated phenol, $250 \mu 1$ of chloroform:isoamylalcohol (24:1) and $250 \mu \mathrm{l}$ of $0.5-\mathrm{mm}$ diameter glass ballontini beads. This mixture was disrupted at maximum speed on a mini-beadbeater (Biospec Products, Stratech Scientific, Luton) for $50 \mathrm{~s}$ and centrifuged at $9500 \mathrm{~g}$ for $5 \mathrm{~min}$. The supernate was precipitated at room temperature for $5 \mathrm{~min}$ with 0.54 volumes of isopropanol and the DNA was pelleted at $9500 \mathrm{~g}$ for $10 \mathrm{~min}$. The pellet was washed in ethanol $70 \% \mathrm{v} / \mathrm{v}$, vacuum dried and resuspended in $200 \mu \mathrm{l}$ of distilled water.

\section{Polymerase chain reaction}

All oligonucleotide primers were obtained from the Advanced Biotechnology Centre, Charing Cross and Westminster Medical School, London and were produced on an Applied Biosystems Model 380B DNA synthesiser (Applied Biosystems, Warrington, Cheshire).

All PCR reactions were performed under the same reaction conditions and cycling times. Each 50- $\mu$ l reaction mix contained $50 \mathrm{mM} \mathrm{KCl}, 10 \mathrm{mM}$ Tris- $\mathrm{HCl}$, $\mathrm{pH} 8.3,1.0 \mathrm{mM} \mathrm{MgCl}_{2}, 20 \mu \mathrm{M}$ each of dATP, dCTP, dGTP dTTP, $2 \mu \mathrm{M}$ of each oligonucleotide primer, 0.5 units of Taq DNA polymerase and c. $25 \mathrm{ng}$ of DNA. The reaction mixtures were amplified (Omnigene, Hybaid, London) as follows: $94^{\circ} \mathrm{C} \times 4 \mathrm{~min}$ (1 cycle), $94^{\circ} \mathrm{C} \times 30 \mathrm{~s}, \quad 66^{\circ} \mathrm{C} \times 90 \mathrm{~s}, \quad 72^{\circ} \mathrm{C} \times 15 \mathrm{~s} \quad(30$ cycles) followed by $72^{\circ} \mathrm{C}$ for $5 \mathrm{~min}$ [19]. After thermal cycling, $15 \mu \mathrm{l}$ of the amplified product was run on an agarose $1.2 \% \mathrm{w} / \mathrm{v}$ gel, with $1 \times \mathrm{TBE}$ as buffer, stained with ethidium bromide, destained with distilled water and visualised under UV light.

DNA sequencing and species-specific PCR primer design

PCR primers corresponding to nucleotides 40-63 (universal forward primer 5'-GCATATCAATAAGCGGAGGAAAAG-3') and 637-654 (universal reverse 
Table 1. Strains and isolates of yeast species used in this study

\begin{tabular}{|c|c|c|c|}
\hline Yeast species & Strain and source & Yeast species & Strain and source \\
\hline C. albicans & $\begin{array}{l}3153(\mathrm{~N}) \\
3302(\mathrm{~N}) \\
3303(\mathrm{~N}) \\
3310(\mathrm{~N}) \\
3363(\mathrm{~N}) \\
\mathrm{cg}^{*}(\mathrm{D}) \\
659 / 92^{*}(\mathrm{C}) \\
4961^{*}(\mathrm{R}) \\
4963^{*}(\mathrm{R})\end{array}$ & C. parapsilosis & $\begin{array}{l}1183^{*}(\mathrm{M}) \\
1524^{*}(\mathrm{M}) \\
1630^{*}(\mathrm{M}) \\
105-1^{*}(\mathrm{Cx}) \\
3104(\mathrm{~N}) \\
3872(\mathrm{~N}) \\
\operatorname{tcdl}^{*}(\mathrm{D}) \\
\operatorname{tcd}^{*}(\mathrm{D}) \\
\operatorname{tcd}^{*}(\mathrm{D})\end{array}$ \\
\hline \multirow[t]{3}{*}{ C. glabrata } & $\begin{array}{l}3309(\mathrm{~N}) \\
3605(\mathrm{~N}) \\
\operatorname{tcd}^{*}(\mathrm{D}) \\
\operatorname{tcd}^{*}(\mathrm{D}) \\
\text { tcd7* (D) } \\
\operatorname{tcd}^{*} \text { (D) } \\
\text { tcd9* (D) } \\
\text { tcd10* (D) } \\
\operatorname{tcd1} 1^{*} \text { (D) }\end{array}$ & & $\begin{array}{l}\text { tcd4* (D) } \\
\text { sa17* (D) } \\
\text { sa19* (D) } \\
\text { sa23* }^{*} \text { (D) } \\
\text { sa25* (D) }^{*} \text { (D) } \\
\text { cpap* }^{*} \text { (D) } \\
674 / 92^{*} \text { (C) } \\
44^{*} \text { (Cx) }\end{array}$ \\
\hline & $\begin{array}{l}\text { tcd12* (D) } \\
\text { cob3* }^{*} \text { (D) } \\
\text { g503* (D) } \\
\text { afc3* (D) } \\
\text { ctb7 }^{*} \text { (D) }\end{array}$ & C. tropicalis & $\begin{array}{l}3111(\mathrm{~N}) \\
3322(\mathrm{~N}) \\
\text { edbb* }^{*}(\mathrm{D}) \\
558 / 92^{*}(\mathrm{C})\end{array}$ \\
\hline & $\begin{array}{l}571 / 92^{*}(\mathrm{C}) \\
625 / 92^{*}(\mathrm{C}) \\
4962^{*}(\mathrm{R}) \\
11088 A^{*}(\mathrm{Cx})\end{array}$ & C. kefyr & $\begin{array}{l}3106(\mathrm{~N}) \\
3234(\mathrm{~N}) \\
624 / 92^{*}(\mathrm{C})\end{array}$ \\
\hline \multirow[t]{7}{*}{ C. krusei } & $\begin{array}{l}3321(\mathrm{~N}) \\
3599(\mathrm{~N})\end{array}$ & C. sake & $\begin{array}{l}3860(\mathrm{~N}) \\
448^{*}(\mathrm{M})\end{array}$ \\
\hline & $\begin{array}{l}\text { jmcga* }^{\text {(D) }} \\
\text { jmcgo* (D) }^{*} \text { (Dr }^{*} \text { (D) }\end{array}$ & C. norvegensis & $\begin{array}{l}3861(\mathrm{~N}) \\
1589^{*}(\mathrm{M})\end{array}$ \\
\hline & $\begin{array}{l}\mathrm{cdb}^{*}(\mathrm{D}) \\
500 / 92^{*}(\mathrm{C})\end{array}$ & C. lipolytica & $717 / 92^{*}(\mathrm{C})$ \\
\hline & $\begin{array}{l}207^{*}(\mathrm{M}) \\
651^{*}(\mathrm{M})\end{array}$ & C. cutaneum & $136 / 92^{*}(C)$ \\
\hline & $\begin{array}{l}807^{*}(\mathrm{M}) \\
827^{*}(\mathrm{M})\end{array}$ & C. lambia & $130^{*}(\mathrm{M})$ \\
\hline & $\begin{array}{l}919^{*}(\mathrm{M}) \\
920^{*}(\mathrm{M})\end{array}$ & C. humicola & $659^{*}(\mathrm{M})$ \\
\hline & $933^{*}(\mathrm{M})$ & C. lusitaniae & $1372^{*}(\mathrm{M})$ \\
\hline \multirow{2}{*}{ Saccharomyces cerevisiae } & $\begin{array}{l}1507^{*}(\mathrm{M}) \\
3178(\mathrm{~N})\end{array}$ & Trichosporon beigelli & $3853(\mathrm{~N})$ \\
\hline & $3275(\mathrm{~N})$ & Cryptococcus neoformans & $\begin{array}{l}3003(\mathrm{~N}) \\
3216(\mathrm{~N}) \\
3218(\mathrm{~N}) \\
3379(\mathrm{~N})\end{array}$ \\
\hline
\end{tabular}

Letters in parentheses indicate source of each isolate as follows: N, National Collection of Pathogenic Fungi, MRL, Bristol; D, Department of Microbiology, Trinity College Dublin, Ireland; C, Department of Microbiology, University of Wales College of Medicine, Cardiff; R, Department of Infectious Diseases and Bacteriology, RPMS, London; M, Department of Infectious Diseases, Monsall Hospital, Manchester; Cx, Department of Medical Microbiology, CXWMS, London.

${ }^{*}$ Clinical isolate.

primer 5'-GGTCCGTGTTTCAAGACG-3') of the Saccharomyces cerevisiae $\mathrm{V} 3$ region of large subunit rDNA have been reported previously [19]. These primers, which are universally conserved within the fungal kingdom, were used to generate PCR products from the following Candida strains: C. albicans NCPF 3302, C. glabrata 11088A, C. parapsilosis 44 and C. krusei 105-1. Amplified PCR products were purified on a Microcon 100 column (Amicon Inc., Beverly, USA) according to the manufacturer's instructions. Sequencing was performed on an Applied Biosystems 373A DNA sequencer with the PRISM ${ }^{\mathrm{TM}}$ Ready Reaction DyeDeoxy ${ }^{\mathrm{TM}}$ Cycle Sequencing Kit (Applied Biosystems) according to the manufacturer's instruc- tions. Universal forward or reverse primers at $0.2 \mu \mathrm{M}$ were used as the sequencing primers and $c .600 \mathrm{ng}$ of PCR product was included in the reaction. Sequence was determined in both directions and repeated on two PCR products obtained from separate amplifications.

Multiple sequence alignments were performed with Clustal V and areas of non-concordance were identified by manual observation [20]. All potential forward PCR primers from the various Candida spp. sequences were determined with MacVector v4.1.4 software (Eastman Kodak IBI, New Haven, CT, USA). Species-specificity of all potential forward primers was tested by the PCR simulation computer pro- 
gramme, Amplify v1.0 (Bill Engels, University of Wisconsin, Genetics, USA).

The following primers that yielded specific products on the simulation programme were chosen for experimental study and were synthesised: $C$. albicans 5'-TTGGAGCGGCAGGATAATGG-3' (Ca1); C. glabrata 5'-GTTTTGCGCCCCTTGCCTCT-3' (Cg-1); C. parapsilosis 5'-GCGGTAGGATAAGTGCAC-3' (Cpa-1) and C. krusei 5'-GACATGGGAATCGCGCACCG-3' (Ck-1).

Universal PCR products obtained from type strains $C$. sake NCPF 3860 and C. norvegensis NCPF 3861, plus those amplified from Manchester strains 448 and 1589 were also sequenced.

\section{Results}

Design of species-specific primers

Approximately $600 \mathrm{bp}$ of DNA from the V3 region was amplified with the universal primers. In all cases the nucleotide sequence was determined in both directions and only overlapping regions were included.

Totals of $502,509,501,518,501,516,516$ and 518 nucleotides were sequenced from the $\mathrm{V} 3$ regions of $C$. albicans NCPF 3302, C. glabrata 11088A, $C$. parapsilosis 44, C. krusei 105-1, C. sake type strain NCPF 3860, C. norvegensis type strain NCPF 3861, Manchester strain 448 and Manchester strain 1589 respectively. Comparison of the sequences obtained from C. albicans NCPF 3302, C. glabrata 11088A, $C$. parapsilosis 44 and $C$. krusei 105-1 revealed differences that were exploited to design primers for the amplification of homologous DNA only. The localisation of these primers within the V3 region of the large subunit rDNA is shown in Fig. 1.
Initially the specificity of the four PCRs was tested with DNA extracted from the four strains from which the primer sequences had been derived. Inclusion of one of the primers, $\mathrm{Ca}-1, \mathrm{Cg}-1, \mathrm{Cpa}-1$ or $\mathrm{Ck}-1$, in a PCR reaction with the universal reverse primer and homologous template DNA yielded the expected amplicons of $156,217,139$ and $226 \mathrm{bp}$, respectively. No amplification was seen when heterologous DNA from the other three strains was included as target (Fig. 2). This confirmed the prediction that $\mathrm{Ca}-1, \mathrm{Cg}-$ 1, Cpa-1 and Ck-1 would be species-specific primers.

\section{Identification of Candida spp. by PCR}

The usefulness of these primers in the identification of various Candida spp. was then examined. DNA extracted from all the yeasts listed in Table 1 was randomly coded by a colleague not involved in this work. DNA from all 82 strains was amplified by each of the primer pairs and designated as $C$. albicans, $C$. glabrata, C. krusei, C. parapsilosis or none of these, i.e., no PCR product with any of the four PCRs. The code was then broken and comparison was made between conventional and PCR identification. The results obtained are summarised in Table 2 .

All C. albicans, C. glabrata, C. krusei and $C$. parapsilosis strains were identified correctly. However, four of 18 of the $C$. krusei strain DNAs yielded weak bands with the $C$. glabrata PCR when $64^{\circ} \mathrm{C}$ was used as the annealing temperature. These bands disappeared when the temperature was increased to $66^{\circ} \mathrm{C}$.

None of the DNA extracts obtained from other yeast genera (Saccharomyces, Cryptococcus or Trichosporon) produced a product with any of the four Candida PCRs. Similarly 15 of 17 other Candida strains (nine species) yielded no PCR products. However, amplification of DNA obtained from two Manchester isolates

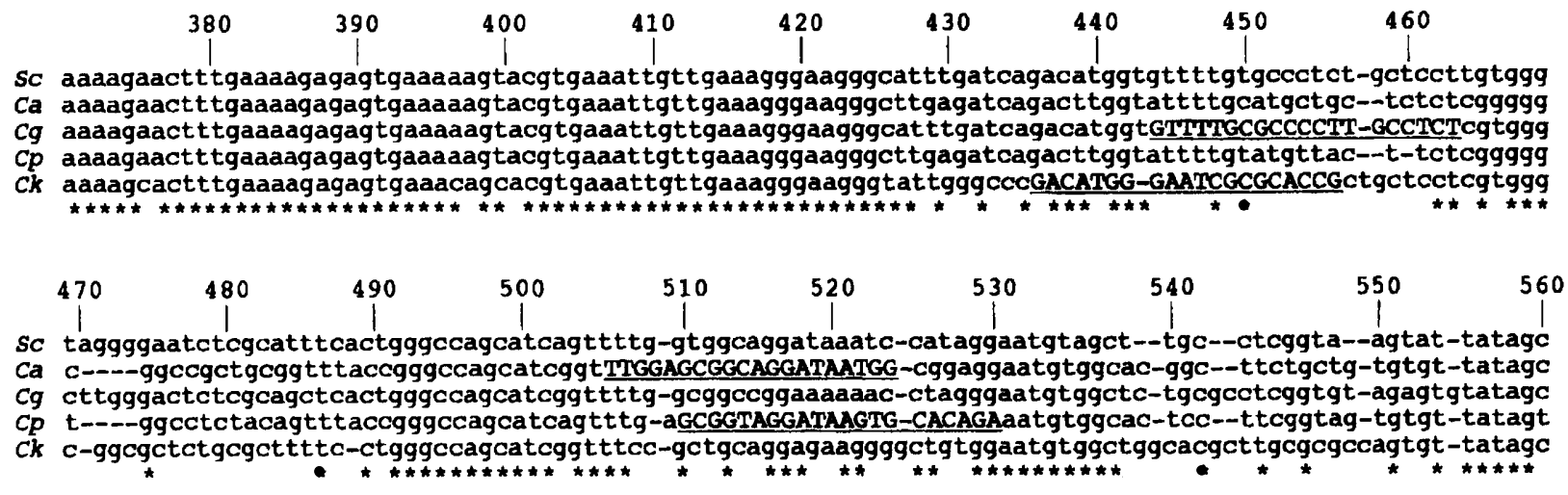

Fig. 1. Partial sequence of the V3 region of large subunit rDNA from: Sc, Saccharomyces cerevisiae (sequence published by Gutell and Fox [25]); Ca, C. albicans NCPF 3302 (Z48339); Cg, C. glabrata 11088A (Z48341); Cp, C. parapsilosis 44 (Z48343) and Ck, C. krusei 105-1 (Z48342). Numbers in parentheses refer to the accession number for each sequence. Figures above the line indicate positions in the $S$. cerevisiae V3 region and the asterisks below indicate conserved nucleotides. Sequences in upper case and underlined are those used as species-specific primers in conjunction with a universal reverse primer [19]. 
A

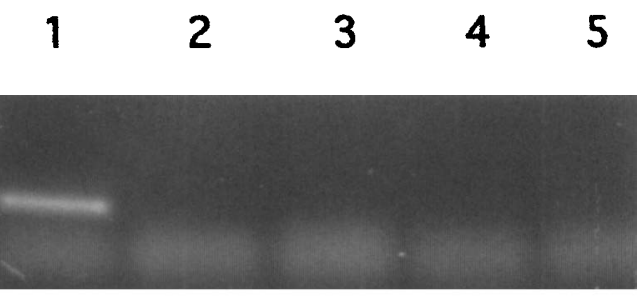

B

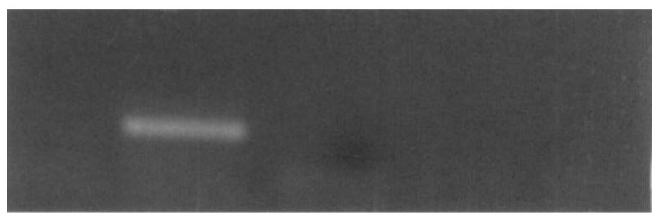

C

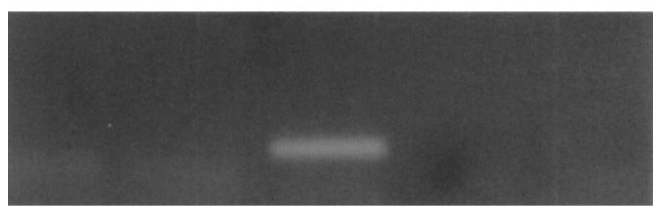

D

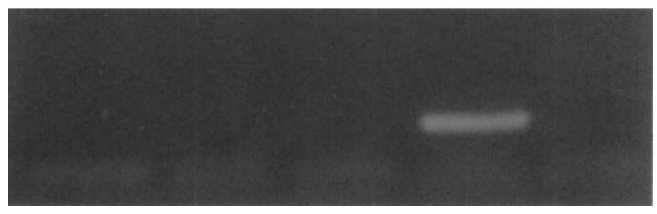

Fig. 2. Agarose gel electrophoresis of PCR products obtained with the universal reverse primer and primers: Ca-1 (panel A); Cg-1 (B); Ca-1 (C) and Ck-1 (D). Target DNA was from $C$. albicans NCPF 3302 (lane 1), $C$. glabrata $11088 \mathrm{~A}(2), C$. parapsilosis 44 (3) and C. krusei 105-1 (4). Material shown in lane 5 was from amplification experiments without target DNA.

identified as C. sake 448 and C. norvegensis 1589 gave strong and reproducible PCR products of the expected sizes with the $C$. albicans and $C$. krusei PCRs, respectively (data not shown).

After the code had been broken each of the 82 strains was cultured again and DNA was extracted. Amplification of these second DNA extractions resulted in $100 \%$ concordance with the results of the blind PCR study.
Human or bacterial DNA purified from clinical isolates of Staphylococcus epidermidis, Staph. aureus, Streptococcus agalactiae, Pseudomonas aeruginosa, Citrobacter freundii, Enterobacter aerogenes, Klebsiella pneumoniae, Proteus mirabilis, Enterococcus faecalis and Escherichia coli did not result in a product when included in three of the species-specific PCRs. A number of weak products were obtained with the $C$. krusei-specific PCR when DNA from Ps. aeruginosa, Cit. freundii or Ent. faecalis was included. However, when the amplifications were repeated no consistency in band pattern was seen and no amplicons of the expected size were seen.

Comparison of the Manchester strain 448, C. sake NCPF 3860 and C. albicans NCPF 3302 rDNA sequences

To determine if the amplification of Manchester strain 448 in the $C$. albicans PCR was a true false positive reaction, its V3 region sequence was determined and compared with those of the $C$. sake type strain (NCPF 3860) and C. albicans NCPF 3302 (the strain used to design the C. albicans-specific primer Ca-1). Seventyfive $b p$ differences were detected in the V3 region between the Manchester strain 448 (Z48566) and $C$. sake NCPF 3860 (Z48580), whereas only $17 \mathrm{bp}$ changes were seen with the sequence of $C$. albicans NCPF 3302.

Amplification of DNA extracted from the C. sake type strain NCPF 3860 , by all four species-specific PCRs, yielded no products (data not shown).

Comparison of the Manchester strain 1589, C. norvegensis NCPF 3861 and C. krusei 105-1 $r D N A$ sequences

Sequencing of the V3 region of Manchester strain 1589 (Z48567) revealed $143 \mathrm{bp}$ changes compared with the $C$. norvegensis type strain NCPF 3861 (Z48568). However, no differences were seen in the sequences of $C$. krusei 105-1 and Manchester strain 1589.

Table 2. Identification of $C$. albicans, C. glabrata, C. parapsilosis and C. krusei by species-specific PCR

\begin{tabular}{|c|c|c|c|c|}
\hline \multirow[b]{2}{*}{ Template DNA (number assayed) } & \multicolumn{4}{|c|}{ Number of strains positive by species-specific PCR for } \\
\hline & C. albicans & C. glabrata & C. parapsilosis & C. krusei \\
\hline C. albicans (9) & 9 & 0 & 0 & 0 \\
\hline C. glabrata (18) & 0 & 18 & 0 & 0 \\
\hline C. parapsilosis (13) & 0 & 0 & 13 & 0 \\
\hline C. krusei (18) & 0 & 0* & 0 & 18 \\
\hline Other Candida spp. $(17) \dagger$ & $1^{\ddagger}$ & 0 & 0 & $0^{\S}$ \\
\hline Other yeast spp. (7) & 0 & 0 & 0 & 0 \\
\hline
\end{tabular}

\footnotetext{
${ }^{*}$ Four of 18 C. krusei strains gave a weak band in the C. glabrata PCR when $64^{\circ} \mathrm{C}$ was the annealing temperature. These bands disappeared when the annealing temperature was increased to $66^{\circ} \mathrm{C}$.

†See Table 1.

¥Product obtained with Manchester strain 448.

\$Product obtained with Manchester strain 1589 which was originally identified as $C$. norvegensis but has been redesignated as $C$. krusei.
} 
Amplification of DNA extracted from the $C$. norvegensis type strain NCPF 3861, by all four speciesspecific PCRs, yielded no products (data not shown).

\section{Discussion}

Candida spp. are now recognised as major pathogens in many different patient populations [21]. Primary culture from specimens takes a minimum of $24 \mathrm{~h}$ and often $48 \mathrm{~h}$. As candidosis can spread rapidly, particularly in immunocompromised patients, prompt and appropriate therapy is required to reduce morbidity and mortality. To achieve this goal, a rapid identification system is required. Currently, biochemical identification systems, especially API $20 \mathrm{C}$ (bioMérieux) predominate. These require a minimum incubation of $24 \mathrm{~h}$, and often $48 \mathrm{~h}$, post-primary isolation to establish identity. This delay can often result in the species not being identified until $96 \mathrm{~h}$ after receipt of a specimen in the diagnostic laboratory. This may have serious consequences for the patient if inappropriate therapy has been initiated. Quick and simple identification procedures, e.g., germ-tube production, are available for C. albicans but not for other pathogenic Candida spp.

A series of PCRs was developed that differs from previously reported methods $[9,12-14,16,18]$ in utilising the large subunit rDNA gene as a target. These PCRs can be used to identify the four major pathogenic Candida spp.: C. albicans, $C$. glabrata, $C$. parapsilosis and $C$. krusei. The forward primers for these four PCRs were designed from variable sequences contained within the V3 region of the large subunit rDNA of each species; the reverse primer used throughout was to a conserved sequence within the same region [19].

Only two discrepancies between conventional identification techniques and the PCR-based system were seen in a blind study of 82 yeast isolates. Manchester strains 448 (identified as C. sake) and 1589 (identified as $C$. norvegensis) yielded PCR products with the $C$. albicans and C. krusei PCRs, respectively. Comparison of the product from strain 448 with that of the C. sake type strain NCPF 3860 revealed $75 \mathrm{bp}$ changes. Similarly, $143 \mathrm{bp}$ differences were demonstrable between the sequences from strain 1589 and $C$. norvegensis type strain NCPF 3861. In a previous study the same V3 region from nine atypical $C$. albicans (C. dubliniensis sp. nov) [22] strains was sequenced and $100 \%$ concordance was obtained. Furthermore, five $C$. albicans sequences from the same region differed by only a single bp (D. J. Sullivan, T. J. Westerneng, K. A. Haynes, D. C. Coleman, unpublished results). These data strongly suggest that strains within a species vary little in this area of the V3 region and, therefore, both of these Manchester strains have been misidentified as $C$. sake (strain 448) and C. norvegensis (strain 1589), respectively. This is not unlikely given the rarity with which they are encountered in the routine diagnostic laboratory. Further evidence for this was provided by the finding that the $C$. sake and $C$. norvegensis type strains, NCPF 3860 and NCPF 3861, did not yield products with any of the species-specific PCRs.

The sequences from strains 448 and 1589 were then compared with those obtained from C. albicans NCPF 3302 and $C$. krusei 105-1, respectively. No bp differences were identified between the sequences from Manchester strain 1589 and C. krusei 105-1. These results strongly suggest that Manchester strain 1589 should be redesignated as $C$. krusei. However, $17 \mathrm{bp}$ differences were observed in the sequences from Manchester strain 448 and C. albicans NCPF 3302. Therefore, it is unlikely that strain 448 is $C$. albicans. However, the sequence was identical to that of the recently described $C$. dubliniensis [22] and strain 448 has subsequently been identified as such. These sequence comparisons indicate that only one of 82 yeast strains tested blind was misidentified, i.e., Manchester strain 448 (C. dubliniensis) as C. albicans.

Although the $C$. krusei PCR non-specifically amplified DNA from several bacteria, this would not interfere with the use of this procedure to identify Candida spp. rapidly from primary isolation plates. It is inconceivable that an experienced microbiologist would confuse colonies of Ps. aeruginosa, Cit. freundii or Ent. faecalis with those of Candida spp. No non-specific products were seen with the $C$. krusei PCR and heterologous yeast DNA (Table 2).

Unlike the cytochrome P-450 lanosterol- $\alpha$-demethylase PCR protocols, the amplifications described here can all be performed under the same cycling conditions. Furthermore, in contrast to methods reported that utilise the small subunit rRNA genes as targets, they do not require subsequent hybridisation or restriction enzyme analysis for species identification. Hence, all four PCRs can be run simultaneously and identification, to the species level, can be achieved within $3 \mathrm{~h}$ of primary isolation - a saving of between 24 and $72 \mathrm{~h}$ on currently employed identification techniques. This reduction in the time required to identify an invading fungus should improve patient outcome, as appropriate antifungal therapy can be initiated earlier, e.g., if fluconazole therapy had been initiated and the yeast was subsequently identified as C. krusei, a species almost universally resistant to fluconazole, therapy could be adapted accordingly after only $24 \mathrm{~h}$. This would have obvious benefits for patient management.

This approach could be extended to other pathogenic Candida spp., and other fungal species, by sequencing of their rDNA regions and the design of species- 
specific primers [23] - an objective which may become a necessity given the increase in the number of fungi reported as pathogenic [24]. However, use of these PCRs in a diagnostic laboratory would require a significant investment in terms of specialised equipment, licencing agreements, staff training and labour costs.

This study demonstrates the utility of large subunit rDNA species-specific PCR for the rapid identification of Candida spp. isolated from clinical specimens. Of the 82 yeasts studied, 58 isolates belonged to the four major pathogenic species, i.e., $C$. albicans, $C$. glabrata, C. parapsilosis and C. krusei. All of these 58 isolates were correctly identified by the speciesspecific PCR in the blinded study. Furthermore, identification was possible within $3 \mathrm{~h}$ of primary isolation, an improvement on current procedures of up to $72 \mathrm{~h}$. Adoption of this technique should significantly improve on the service currently offered by diagnostic laboratories for identification of Candida spp.

This work was supported in part by The Westminster Hospital Research Trustees, T. W. is in receipt of bursaries from Netherlands Universities Foundation for International Co-operation and Rijks Instituut voor Volksgezondheid en Milieuhygiene, Netherlands. We are grateful to Drs R. A. Barnes, C. K. Campbell, D. W. Denning, D. J. Sullivan and D. W. Warnock and to Professor T. R. Rogers for supplying us with strains.

\section{References}

1. Stein DK, Sugar AM. Fungal infections in the immunocompromised host. Diagn Microbiol Infect Dis 1989; 12: 221S$228 \mathrm{~S}$.

2. Fraser VJ, Jones M, Dunkel J, Storfer S, Medoff G, Dunagan WC. Candidemia in a tertiary care hospital: epidemiology, risk factors, and predictors of mortality. Clin Infect Dis 1992; 15: 414-421.

3. Powderly WG. Mucosal candidiasis caused by non-albicans species of Candida in HIV-positive patients. AIDS 1992; 6, 604-605.

4. Odds FC. Antifungal susceptibility testing of Candida spp. by relative growth measurement at single concentrations of antifungal agents. Antimicrob Agents Chemother 1992; 36: 1727-1737.

5. Fenn JP, Segal H, Barland B et al. Comparison of updated Vitek yeast biochemical card and API 20C yeast identification systems. J Clin Microbiol 1994; 32, 1184-1187.

6. Land GA, Harrison BA, Hulme KL, Cooper BH, Byrd JC. Evaluation of the new API 20C strip for yeast identification against a conventional method. J Clin Microbiol 1979; 10: 357-364.

7. Nickerson WJ. Reduction of inorganic substances by yeasts. I. Extracellular reduction of sulfite by species of Candida. $J$ Infect Dis 1953; 93: 43-56.
8. Odds FC, Bernaerts R. CHROMagar Candida, a new differential isolation medium for presumptive identification of clinically important Candida species. J Clin Microbiol 1994; 32: $1923-1929$.

9. Niesters HGM, Goessens WHF, Meis JFMG, Quint WGV. Rapid, polymerase chain reaction-based identification assays for Candida species. J Clin Microbiol 1993; 31: 904-910.

10. Sullivan D, Bennett D, Henman $\mathrm{M}$ et al. Oligonucleotide fingerprinting of isolates of Candida species other than $C$. albicans and of atypical Candida species from human immunodeficiency virus-positive and AIDS patients. $J$ Clin Microbiol 1993; 31: 2124-2133.

11. Stockman L, Clark KA, Hunt JM, Roberts GD. Evaluation of commercially available acridinium ester-labeled chemiluminescent DNA probes for culture identification of Blastomyces dermatitidis, Coccidioides immitis, Cryptococcus neoformans, and Histoplasma capsulatum. J Clin Microbiol 1993; 31: 845850.

12. Buchman TG, Rossier M, Merz WG, Charache P. Detection of surgical pathogens by in vitro DNA amplification. Part 1 . Rapid identification of Candida albicans by in vitro amplification of a fungus-specific gene. Surgery 1990; 108: 338-347.

13. Crampin AC, Matthews RC. Application of the polymerase chain reaction to the diagnosis of candidosis by amplification of an HSP 90 gene fragment. J Med Microbiol 1993; 39: 233238.

14. Kan VL. Polymerase chain reaction for the diagnosis of candidemia. J Infect Dis 1993; 168: 779-783.

15. Holmes AR, Cannon RD, Shepherd MG, Jenkinson HF Detection of Candida albicans and other yeasts in blood by PCR. J Clin Microbiol 1994; 32: 228-231.

16. Barns SM, Lane DJ, Sogin ML, Bibeau C, Weisburg WG. Evolutionary relationships among pathogenic Candida species and relatives. $J$ Bacteriol 1991; 173: 2250-2255.

17. Maiwald M, Kappe R, Sonntag HG. Rapid presumptive identification of medically relevant yeasts to the species level by polymerase chain reaction and restriction enzyme analysis. J Med Vet Mycol 1994; 32: 115-122.

18. Burgener-Kairuz P, Zuber J-P, Jaunin P, Buchman TG, Bille J, Rossier M. Rapid detection and identification of Candida albicans and Torulopsis (Candida) glabrata in clinical specimens by species-specific nested PCR amplification of a cytochrome P-450 lanosterol- $\alpha$-demethylase (L1A1) gene fragment. J Clin Microbiol 1994; 32: 1902-1907.

19. Fell JW. Rapid identification of yeast species using three primers in a polymerase chain reaction. Mol Marine Biol Biotechnol 1993; 2: 174-180.

20. Higgins DG, Sharp PM. Fast and sensitive multiple sequence alignments on a microcomputer. Comput Appl Biosci 1989; 5: 151-153.

21. Musial CE, Cockerill FR, Roberts GD. Fungal infections in the immunocompromised host: clinical and laboratory aspects. Clin Microbiol Rev 1988; 1: 349-364.

22. Sullivan DH, Westerneng TJ, Haynes KA, Bennet DE, Coleman DC. Candida dubliniensis sp. nov., phenotypic and molecular characterization of a novel species associated with oral candidosis in HIV-infected individuals. Microbiol 1995; 141: $1507-1521$.

23. Haynes KA, Westerneng TJ, Fell JW, Moens W. Rapid detection and identification of pathogenic fungi by PCR amplification of large subunit ribosomal DNA. J Med Vet Mycol 1995; 33: 319-326.

24. Anaissie E, Bodey GP, Kantarjian $\mathrm{H}$ et al. New spectrum of fungal infections in patients with cancer. Rev Infect Dis 1989; 11: $369-378$.

25. Gutell RR, Fox GE. Compilation of large subunit RNA sequences presented in a structural format. Nucleic Acids Res 1988; 16 (Suppl): r175-r269. 